\title{
Influencing Factors of HLA-DR Expression in Orbital Preadipocytes of Patients With Thyroid Associated Ophthalmopathy
}

\section{Xiang Lei}

Central South University Third Xiangya Hospital

Hanhan Liu

Central South University Third Xiangya Hospital

Hong Ren

Central South University Third Xiangya Hospital

\section{Xueliang Xu}

Central South University First Hospital

Zhihong Deng ( $\nabla$ dzhxy3yy@126.com )

Central South University Third Xiangya Hospital

\section{Research Article}

Keywords: thyroid-associated ophthalmopathy, preadipocytes, HLA-DR, IFN- $y$, IL-6

Posted Date: August 31st, 2021

DOI: https://doi.org/10.21203/rs.3.rs-845021/v1

License: (9) This work is licensed under a Creative Commons Attribution 4.0 International License. Read Full License 


\section{Abstract}

Purpose: To investigate the effects of IFN- - , IL-6, PPAR-y agonist pioglitazone and PPAR- $y$ antagonist GW9662 on preadipocyte proliferation and HLA-DR expression.

Methods: Preadipocytes were cultured from thyroid associated ophthalmopathy patients and health ocular trauma patients and induced to differentiate in vitro. IFN-y, IL-6, PPAR-y agonist pioglitazone and PPAR-y antagonist GW9662 were added to preadipocytes to observe their effects on proliferation and HLA-DR expression.

Results: IFN-y significantly increased the levels of HLA-DR mRNA and protein in preadipocytes which are cultured from thyroid associated ophthalmopathy patients. IFN-y also reduced the accumulation of lipid in the cytoplasm of preadipocytes. On the contrary, IL- 6 had no effect on the expression of HLA-DR. Pioglitazone increased the expression of HLA-DR in orbital preadipocytes of TAO patients, and promoted the proliferation and intracellular lipid accumulation of preadipocytes. On the contrary, GW9662 decreased the expression of HLA-DR in orbital preadipocytes of TAO patients, and reduced the proliferation and intracellular lipid accumulation of preadipocytes.

Conclusion: IFN-y increase the expression of HLA-DR in TAO orbital preadipocytes after differentiation. PPAR - $Y$ antagonists may be beneficial to the treatment of TAO.

\section{Introduction}

Thyroid-associated ophthalmopathy (TAO) is usually part of a systemic process in patients with hyperthyroidism in which the auto-antibodies target the eyes, thyroid and skin. The annual incidence of TAO is around $16 / 100,000$ in women and $3 / 100,000$ in men ${ }^{[1]}$. There is no effective medication for TAO due to its poorly understand pathogenesis. Orbital inflammatory reaction is one of the key pathological factors underlying the symptoms and signs of TAO. Its initial stage is characterized by the increase of lymphocyte activity and the production of IL-1 $\beta \otimes$ IFN- $\gamma$ Isocytokines. While IL-1 $\beta$ can stimulate increase IL-6 production in orbital fibroblasts ${ }^{[1]}$. In the orbit of TAO patients, the over activation of preadipocytes is the main reason for the excessive volume of orbital adipose tissue and the increase of orbital pressure- $\gamma$, which plays a key role in driving the differentiation of preadipocytes ${ }^{[2]}$. Human leukocyte DR antigen (HLA-DR) is the major histocompatibility antigen involved in the orbital inflammation of TAO patients. Immunohistochemical analysis has demonstrated wide expression patterns of HLA-DR involved in the orbital inflammation of TAO, in retrobulbar connective tissue, extraocular muscle and fibroblast cells of TAO patients ${ }^{[3-5]}$. In the orbit of TAO patients, over-activated adipogenesis of preadipocytes is a major contributor to excess orbital adipose tissue volume and increased orbital pressure. PPAR-y plays a key role in driving the differentiation of preadipocytes ${ }^{[2]}$. Our previous study found that the expression of HLADR in preorbital adipocytes of TAO patients was significantly higher than that of normal people ${ }^{[2]}$. In order to further understand the influencing factors of HLA-DR expression in preorbital adipocytes of TAO patients. In this study, the orbital preadipocytes of TAO patients and normal people were cultured in vitro. 
IFN- $\gamma$, PPAR- $\gamma$, IL-6, PPAR- $\gamma$ Agonists pioglitazone and antagonist GW9662 were added to the cultured orbital preadipocytes. The preadipocyte differentiation and HLA-DR expression were observed.

\section{Materials And Methods}

\section{In-vitro culture and differentiation of preadipocytes}

The study was approved by the Institutional Review Boards of Xiangya 3th Hospital of Central-south Uniersity (Changsha, Hunan, China) and written informed consent was obtained from each subject. Orbital adipose/connective tissues of 5 patients with TAO underwent orbital decompression were obtained, including 3 females and 2 males; 37-52 years old (mean age 45 years old), inclusion criteria: 1) meet the Tao diagnostic criteria, 2) have normal thyroid function for more than 6 months, 3) have never received iodine 131 treatment or orbital radiotherapy. Normal orbital adipose tissues were obtained from four patients who underwent ophthalmic surgery due to trauma, including 2 females and 2 males; 38-45 years old (average age 42 years old). The inclusion criteria of the control group were: 1 ) no autoimmune disease, 2) no history of thyroid disease or orbital inflammation disorders.

Orbital tissues were minced and placed directly in plastic culture dishes, allowing preadipocytes to proliferate. Cells were cultured with DMEM /F-12(1:1) containing 20\% FBS, penicillin (100 U/mL) and streptomycin $(100 \mathrm{U} / \mathrm{mL})$ in a humidified $5 \% \mathrm{CO} 2$ incubator at $37^{\circ} \mathrm{C}$. The preadipocyte differentiation was induced as follows. When orbital cells reached confluence after in-vitro culture for 3-5 days, the culture medium were replaced by serum-free DMEM/F12 (1:1) supplemented with biotin (33 mmol/L), pantothenic acid $(17 \mathrm{mmol} / \mathrm{L})$, transferrin $(10 \mathrm{mg} / \mathrm{mL})$, triiodothyronine $(0.2 \mathrm{nmol} / \mathrm{L})$, insulin $(1 \mathrm{mmol} / \mathrm{L})$, and (for the first 4 days only) dexamethasone $(1 \mu \mathrm{mol} / \mathrm{L})$ and isobutylmethylxanthine (IBMX; 0.1 $\mathrm{mmol} / \mathrm{L}$ ). The differentiation continued for 13 days and the medium were replaced every $3-4$ days.

To evaluate the effect of cytokines on preadipocyte differentiation and HLA-DR expression, cell cultures were treated with IFN-y $(1 \mathrm{ng} / \mathrm{ml}$, SIGMA $), I L-6(1 \mathrm{ng} / \mathrm{ml}$, SIGMA), PPAR-yagonist pioglitazone $(10 \mu \mathrm{mol} / \mathrm{L})$ or PPAR-yantagonist GW9662 $(10 \mu \mathrm{mol} / \mathrm{L})$ and cultured for 13 days. The morphological changes of TAO and normal preorbital adipocytes after differentiation were observed.

\section{Oil Red 0 staining}

After differentiation, cells were washed with $1 \times$ PBS, fixed in $10 \%$ formalin overnight at room temperature and rinsed in distilled water. Cells were stained with filtered $0.21 \%$ Oil Red $\mathrm{O}$ for $2 \mathrm{~h}$ and then again rinsed in distilled water. Then cells were exposed to isopropanol to eliminate extra dye, rinsed with PBS and visualized under a microscope.

\section{Intracytoplasmic lipid (triglyceride accumulation) detection}

Cells were grown in 96-well plates for density of (3-5) $\times 10^{7} / \mathrm{L}$. After differentiation, cells were fixed in $10 \%$ formalin for $1 \mathrm{~h}$, and rinsed with distilled water. Cells were stained with filtered $0.21 \%$ Oil Red 0 for $2 \mathrm{~h}$ 
and then rinsed in distilled water for several times. Isopropanol was added to extract the dye after water was vaporized in the incubator. The absorbance (OD value) was measured on wavelength of $510 \mathrm{~nm}$ using the ultraviolet spectrophotometer.

\section{Flow Cytometry}

Cells were harvested using $0.25 \%$ trypsin/ EDTA and fixed for $30 \mathrm{~min}$ in ice-cold $2 \%$ formaldehyde. The fixed cells were washed in flow cytometry buffer (PBS, $2 \%$ FBS, $0.2 \%$ Tween 20 ) and incubated for 20 min in flow cytometry buffer containing PE-conjugated monoclonal antibodies against CD29 (eBioscience, Clone: TS2/16.2.1), CD44 (eBioscience, Clone: P2A1), CD49d (eBioscience, Clone: BU49) or HLA-DR (eBioscience, Clone: JM-DR), respectively. Control experiments include blank and isotype IgG. The stained cells were analyzed by BD FACSCalibur.

\section{MTT colorimetric assay}

Cells were collected and adjust the cell density to $5 \times 104$ / L, inoculated on six well plate, cultured for 24 hours. $10 \mu \mathrm{mol} / \mathrm{L}$ pioglitazone and GW9662 were added. After 48 hours of continuous culture, 20ul 5mg / ml MTT solution was added to each well. After 4 hours of culture, the supernatant was discarded, 100ul DMSO was added to fully dissolve, and the absorbance (OD value) was measured at the wavelength of 570nm with an enzyme labeling instrument.

\section{Immunohistochemistry}

Cells were grown in six-well plates with coverslips. HLA-DR expression was measured using the SP histological assay and HLA-DR antibody (abcam, ab20181). Brown dye in cytoplasm or cell membrane was found in HLA-DR positive cells.

\section{Quantitative Real-time RT-PCR}

Total RNA was isolated from cell cultures using Trizol and reverse transcribed into cDNA. Primers were designed using primer premier 5.0 and the sequences were as follows: HLA-DR, 5'- aggcgagtttatgtttg - $3^{\prime}$ (sense) and 5'- cagggctgttcgtgag -3' (antisense). GAPDH, 5'-aatcccatcaccatcttcc-3' (sense) and 5'catcacgccacagtttcc-3' (antisense). Quantitative real-time PCR was performed using SYBR Green method on LightCycler system. The relative expression levels of HLA-DR were calculated with the 2- $\Delta \Delta \mathrm{Ct}$ formula ${ }^{[6]}$.

\section{Statistical analysis}

The SPSS software package (version 11.0) was used for the statistical analysis. The data were from at least three separate experiments and expressed as mean $\pm S$.D. Two-sided non-paired t-test was used to analyze the data from two groups. Statistical differences were considered significant when $p<0.05$.

\section{Results}




\section{In-vitro culture and differentiation of preadipocytes}

Under standard differentiation conditions, cell proliferation ceased and cells displayed dipogenic phenotypes. The cells lost their stellate morphology at one day after differentiation and then converted to an oval or spherical shape (Fig.1A). Oil Red 0 staining showed that the discrete lipid vacuoles began to appear in cytoplasm after 6 days and gradually increased in number and size throughout the entire culture period (Fig.1B). It suggests that the in-vitro cultured preadipocytes could differentiate into mature adipocytes.

\section{HLA-DR expression in orbital preadipocytes from TAO patients}

The immunohistochemical analysis showed that HLA-DR was expressed at much higher level in orbital preadipocytes from TAO patients compared to controls (Fig.2A), and HLA-DR was mainly localized in cytoplasm and cell membrane. HLA-DR expression in orbital preadipocytes was further confirmed by flow cytometry and the results showed that HLA-DR expression in TAO orbital preadipocytes was further enhanced after they were differentiated into mature adipocytes while control orbital preadipocytes had no detectable HLA-DR expression even after differentiation (Fig.2B). Consistently, real-time PCR (Fig.2C) and immunoblot (Fig.2D) results showed that HLA-DR expression level was increased in TAO orbital preadipocytes after differentiation.

\section{Effects of IFN-y or IL-6 on preadipocyte differentiation and HLA-DR expression}

The results showed that the percentage of cells containing lipid droplets was reduced in IFN-y treated TAO preadipocytes while IL-6 treatment had no such effect (Fig.3A). Consistently, IFN- $y$ also reduced intracytoplasmic lipid accumulation in TAO preadipocytes (Fig.3B). Thus, IFN- - , but not IL-6, reduced the number of cells undergoing adipogenesis and the degree of triglyceride accumulation.

The effects of IFN- $y$ or IL- 6 on HLA-DR expression in orbital cells from TAO patients were assessed by quantitative real-time RT-PCR and immunoblot after 13 days of differentiation and co-treatment with IFNy or IL-6. The results showed that HLA-DR mRNA (Fig.3C) and protein (Fig.3D) levels were greatly increased by IFN- $y$ treatment in TAO preadipocytes. In contrast, IL- 6 treatment could not change the HLADR expression levels.

\section{The effects of PPAR-y agonist and antagonist on preadipocytes}

MTT assay showed that pioglitazone promoted preadipocyte proliferation does-dependently while GW9662 inhibited preadipocyte proliferation does-dependently (Fig.4A). Oil Red 0 staining showed that the percentage of cells containing lipid droplets was increased by pioglitazone in a does-dependent manner (Fig.4B). In contrast, GW9662 reduced lipid droplets in a does-dependent manner.

We further investigated the effects of pioglitazone or GW9662 on HLA-DR expression in preadipocytes. Quantitative real-time PCR (Fig.4C) and immunoblot (Fig.4D) showed that pioglitazone increased HLA-DR 
expression in orbital preadipocytes from TAO patients. In contrast, GW9662 reduced the expression of HLA-DR in orbital preadipocytes from TAO patients.

\section{Discussion}

TAO is an autoimmune disease with lymphocytes activated by autoantigens within thyroid gland and with autoantibodies directed against the shared antigen in the orbit ${ }^{[7]}$. Lymph-drainage and the immune cells which present antigens to immune system are absent in the orbit of normal subjects. Therefore, the antigen presenting cells (APC) in the orbit is dispensable for the pathogenesis of TAO. MHC class-II molecules are critical for the function of APC. HLA-DR is one of the most important MHC class-II antigens. Early studies demonstrate that a large number of cells express HLA-DR in extraocular muscle interstitial and adipose /connected tissues from TAO patients ${ }^{[3,4]}$. Agretti $P$ et al showed that orbital preadipocytes in TAO express TSH receptor ${ }^{[8]}$, the major target antigens in the orbit. Meanwhile, enhanced adipogenesis are found in the orbit of $\mathrm{TAO}^{[9]}$. Here, we show that HLA-DR is expressed in orbital preadipocytes from TAO patients, but not healthy adults, by flow cytometry and immunohistochemistry. These results were consistent with previous studies ${ }^{[10-12]}$. It's possible that HLA-DR positive adipocytes in the orbit act as APC and present orbital autoantigens to immune system, accelerating attack from immune reactions.

Inflammation-related cytokines was dys-regulated in the autoimmune thyroid gland and orbital tissues in $\mathrm{TAO}^{[13,14]}$. They act as soluble signals among cells to promote autoimmune reactions through activating T and B lymphocytes ${ }^{[15,16]}$. Th1-type cytokines, such as IFN-Y, IL-1, IL-2, TGF- $\beta$ and TNF- $\alpha$, are dominant in the early stage of TAO and they could induce or amplify inflammation in the orbit ${ }^{[17,18]}$. In the late (chronic and stable) stage, Th2-type cytokines, such as IL-4, IL-5, IL-6, and IL-10, are dominant and they could promote fibrosis of orbit tissue ${ }^{[19,20]}$. In our study, we treated orbital preadipocytes from TAO patients and healthy adults with IFN-y or IL-6 which represents Th1-type or Th2-type cytokines, respectively. We found that IFN- $\gamma$, but not IL-6, inhibits adipogenesis. This result was supported by Valyasevi et al ${ }^{[21]}$. The mechanisms by which IFN-y affects adipocyte differentiation may involve downregulation of lipoprteinlipase (LPL), peroxisome proliferator-activated receptor-y 2 (PPAR-y 2) ${ }^{[23]}$. Since PPAR-y played a key role in preadipocyte proliferation, we continued to study the effect of PPAR- $\gamma$ agonists pioglitazone and antagonist GW9662 on preadipocyte proliferation. The results showed that pioglitazone promoted the proliferation of preadipocytes, while GW9662 inhibited it. PPAR- $\gamma$ Antagonists may be beneficial to the treatment of TAO.

HLA-DR up-regulation in orbital preadipocytes from TAO patients after differentiation may amplify the inflammatory reaction in orbit, enhance the activity of immune cells and promote the process of presenting autoantigens. These pro-inflammation effects might be exacerbarated by preadipocytes proliferation ${ }^{[22]}$. Quantitative real-time RT-PCR shows that HLA-DR expression in orbital preadipocytes from TAO patients is much higher after differentiation and IFN- $y$ can further increase HLA-DR expression. It supports that IFN-y plays a vital role in the pathogenesis of TAO and Th1-type cytokine is one of the 
important factors in developing and maintaining the course of TAO. Thus, early intervention with

anticytokine agents might be useful in clinic ${ }^{[22]}$. Another important finding in our study is that GW9662, a PPAR- $\gamma$ antagonist, inhibited preadipocyte proliferation and reduced lipid droplets and HLA-DR expression. It suggests that GW9662 might have therapeutic potential in TAO patients.

In summary, we report that IFN-y promoted the differentiation and HLA-DR expression in preadipocytes, while IL-6 don't. PPAR-y agonist GW9662 inhibited preadipocyte proliferation. Thus, PPAR-y antagonist might be beneficial for TAO treatment.

\section{Declarations}

\section{Conflict of Interest Statement}

The authors declare that they have no conflict of interest.

\section{References}

1. Hiromatsu Y, Eguchi H, Tani J, Kasaoka M, Teshima Y. (Tokyo (2014) Graves' ophthalmopathy: epidemiology and natural history. Internal medicine. Japan) 53(5):353-360. https://doi.org/10.2169/internalmedicine.53.1518

2. Yang W, Yang C, Luo J, Wei Y, Wang W, Zhong Y (2018) Adiponectin promotes preadipocyte differentiation via the PPARY pathway. Mol Med Rep 17(1):428-435. https://doi.org/10.3892/mmr.2017.7881

3. Tallstedt L, Norberg R (1988) Immunohistochemical staining of normal and Graves' extraocular muscle. Investig Ophthalmol Vis Sci 29(2):175-184

4. Weetman AP, Cohen S, Gatter KC, Fells P, Shine B (1989) Immunohistochemical analysis of the retrobulbar tissues in Graves' ophthalmopathy. Clin Exp Immunol 75(2):222-227

5. Nair S, Lee YH, Rousseau E, Cam M, Tataranni PA, Baier LJ, Bogardus C, Permana PA (2005) Increased expression of inflammation-related genes in cultured preadipocytes/stromal vascular cells from obese compared with non-obese Pima Indians. Diabetologia 48(9):1784-1788. https://doi.org/10.1007/s00125-005-1868-2

6. Livak KJ, Schmittgen TD (2001) Analysis of relative gene expression data using real-time quantitative PCR and the 2(-Delta Delta C(T)) Method. Methods (San Diego, Calif.), 25(4), 402-408. https://doi.org/10.1006/meth.2001.1262

7. Weetman AP (1991) Thyroid-associated eye disease: pathophysiology[J]. Lancet 338(8758):25-28

8. Agretti P, De Marco G, De Servi M, Marcocci C, Vitti P, Pinchera A, Tonacchera M (2005) Evidence for protein and mRNA TSHr expression in fibroblasts from patients with thyroid-associated ophthalmopathy (TAO) after adipocytic differentiation. European journal of endocrinology 152(5):777-784. https://doi.org/10.1530/eje.1.01900 
9. Kumar S, Coenen MJ, Scherer PE, Bahn RS (2004) Evidence for enhanced adipogenesis in the orbits of patients with Graves' ophthalmopathy. J Clin Endocrinol Metab 89(2):930-935. https://doi.org/10.1210/jc.2003-031427

10. Zuk PA, Zhu M, Ashjian P, De Ugarte DA, Huang JI, Mizuno H, Alfonso ZC, Fraser JK, Benhaim P, Hedrick MH (2002) Human adipose tissue is a source of multipotent stem cells. Molecular biology of the cell 13(12):4279-4295. https://doi.org/10.1091/mbc.e02-02-0105

11. Katz AJ, Tholpady A, Tholpady SS, Shang H, Ogle RC (2005) Cell surface and transcriptional characterization of human adipose-derived adherent stromal (hADAS) cells. Stem cells (Dayton. Ohio) 23(3):412-423. https://doi.org/10.1634/stemcells.2004-0021

12. Kern S, Eichler H, Stoeve J, Klüter H, Bieback K (2006) Comparative analysis of mesenchymal stem cells from bone marrow, umbilical cord blood, or adipose tissue. Stem Cells 24(5):1294-1301. https://doi.org/10.1634/stemcells.2005-0342

13. Mariotti S, Prete GD, Chiovato L, Mastromauro C, Marcocci C, Carli MD, et al (1992) Cytokines and thyroid autoimmunity. International Journal of Immunopathology Pharmacology 5(2):103-113

14. Kumar S, Bahn RS (2003) Relative overexpression of macrophage-derived cytokines in orbital adipose tissue from patients with graves' ophthalmopathy. J Clin Endocrinol Metab 88(9):42464250. https://doi.org/10.1210/jc.2003-030380

15. Rasmussen AK (2000) Cytokine actions on the thyroid gland. Danish medical bulletin 47(2):94-114

16. Ajjan RA, Watson PF, Weetman AP (1996) Cytokines and thyroid function. Adv Neuroimmunol 6(4):359

17. Yang D, Hiromatsu Y, Hoshino T, Inoue Y, Itoh K, Nonaka K (1999) Dominant infiltration of T(H)1-type CD4 + T cells at the retrobulbar space of patients with thyroid-associated ophthalmopathy. Thyroid: official journal of the American Thyroid Association 9(3):305-310. https://doi.org/10.1089/thy.1999.9.305

18. Avunduk AM, Avunduk MC, Pazarli H, Oguz V, Varnell ED, Kaufman HE, Aksoy F (2005) Immunohistochemical analysis of orbital connective tissue specimens of patients with active Graves ophthalmopathy. Curr Eye Res 30(8):631-638. https://doi.org/10.1080/02713680591005931

19. Förster G, Otto E, Hansen C, Ochs K, Kahaly G (1998) Analysis of orbital T cells in thyroid-associated ophthalmopathy. Clin Exp Immunol 112(3):427-434. https://doi.org/10.1046/j.13652249.1998.00613.x

20. Wakelkamp IM, Bakker O, Baldeschi L, Wiersinga WM, Prummel MF (2003) TSH-R expression and cytokine profile in orbital tissue of active vs. inactive Graves' ophthalmopathy patients. Clin Endocrinol 58(3):280-287. https://doi.org/10.1046/j.1365-2265.2003.01708.x

21. Valyasevi RW, Jyonouchi SC, Dutton CM, Munsakul N, Bahn RS (2001) Effect of tumor necrosis factor-alpha, interferon-gamma, and transforming growth factor-beta on adipogenesis and expression of thyrotropin receptor in human orbital preadipocyte fibroblasts. J Clin Endocrinol Metab 86(2):903-908. https://doi.org/10.1210/jcem.86.2.7188 
22. Nobusue H, Kano K (2010) Establishment and characteristics of porcine preadipocyte cell lines derived from mature adipocytes. Journal of cellular biochemistry 109(3):542-552. https://doi.org/10.1002/jcb.22431

23. Hiromatsu Y, Yang D, Miyake I, Koga M, Kameo J, Sato M, Inoue Y, Nonaka K (1998) Nicotinamide decreases cytokine-induced activation of orbital fibroblasts from patients with thyroid-associated ophthalmopathy. J Clin Endocrinol Metab 83(1):121-124. https://doi.org/10.1210/jcem.83.1.4478

24. Paridaens D, van den Bosch WA, van der Loos TL, Krenning EP, van Hagen PM (2005) The effect of etanercept on Graves' ophthalmopathy: a pilot study. Eye 19(12):1286-1289. https://doi.org/10.1038/sj.eye.6701768

\section{Figures}

A

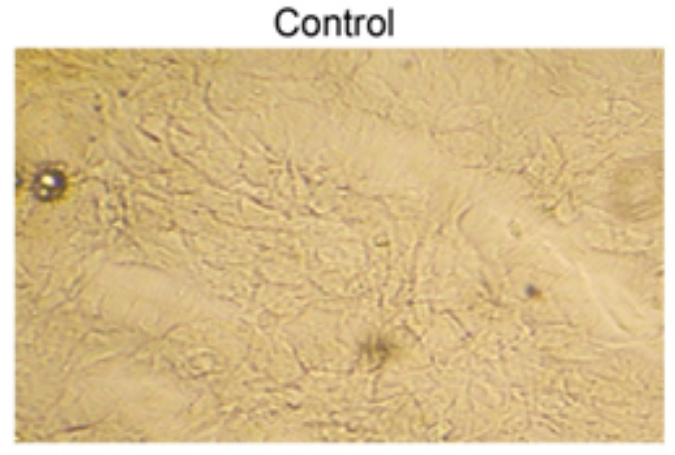

TAO

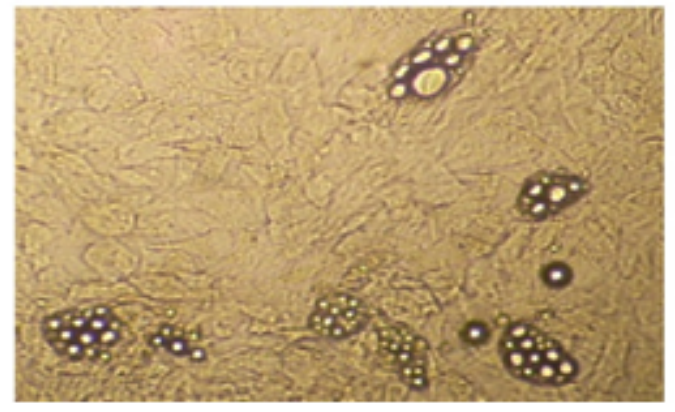

B

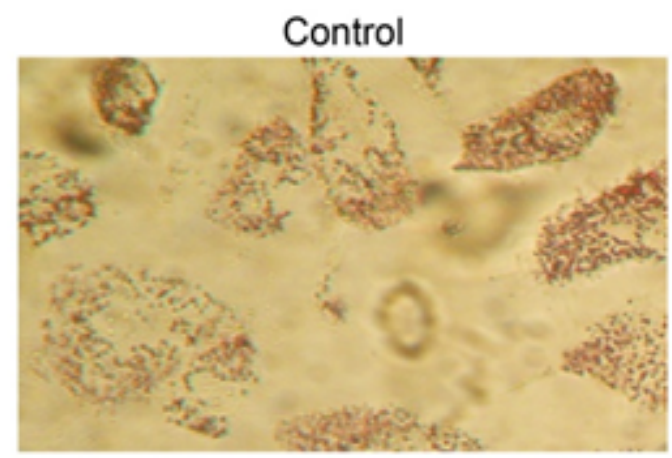

TAO

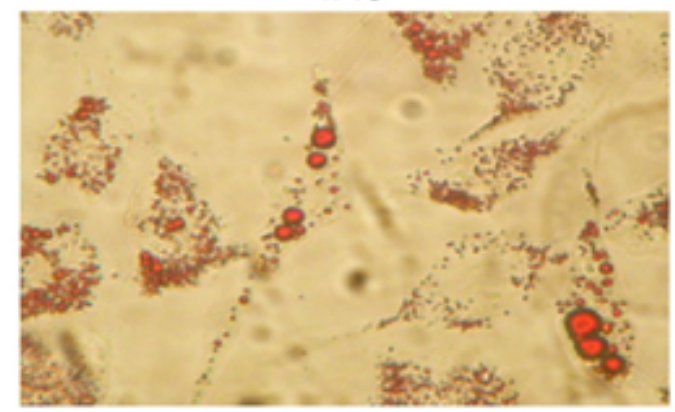

\section{Figure 1}

In-vitro culture and differentiation of preadipocytes from TAO patients $(\times 250)(A)$ The morphology of preadipocytes from TAO patients and controls after differentiation. (B) Oil Red $O$ staining of preadipocytes from TAO patients and controls after differentiation. 
A
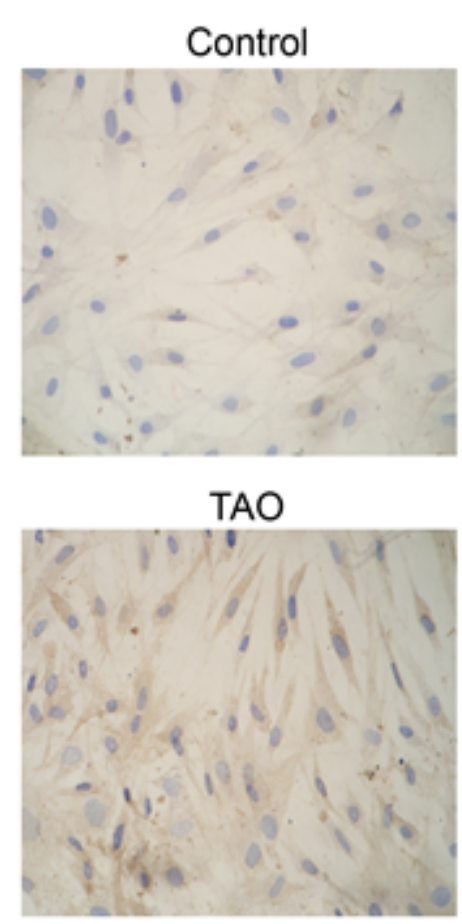

C

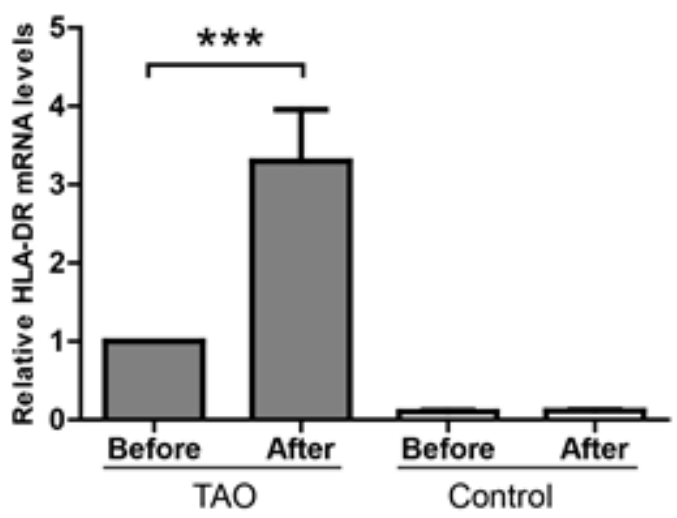

B
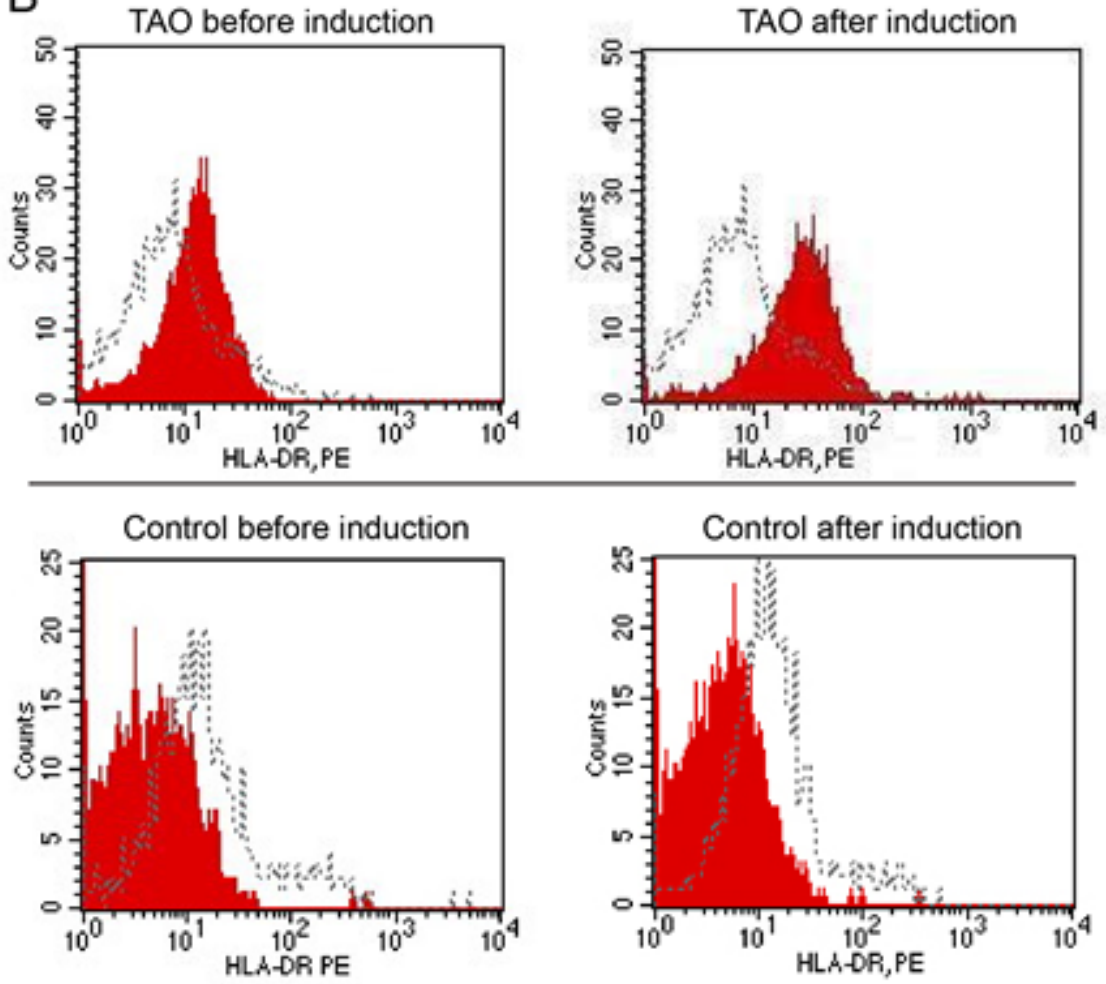

D

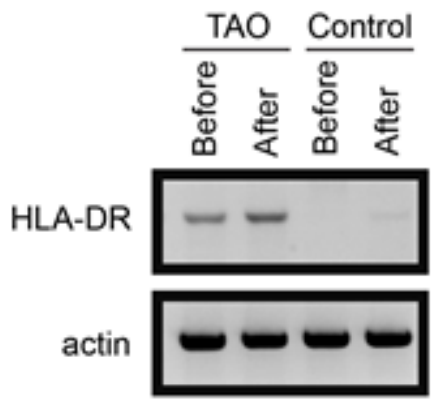

Figure 2

HLA-DR expression in preadipocytes from TAO patients (A) Immunohistochemistry showing HLA-DR expression in differentiated preadipocytes from TAO patients and controls. (B) Flow cytometry showing the staining of HLA-DR in preadipocytes from TAO patients and controls before and after differentiation. (C) Real time-PCR showing the HLA-DR mRNA level in preadipocytes from TAO patients and controls before and after differentiation. (D) Immunoblot showing the HLA-DR protein level in preadipocytes from TAO patients and controls before and after differentiation. 
A

IFN- $\gamma$
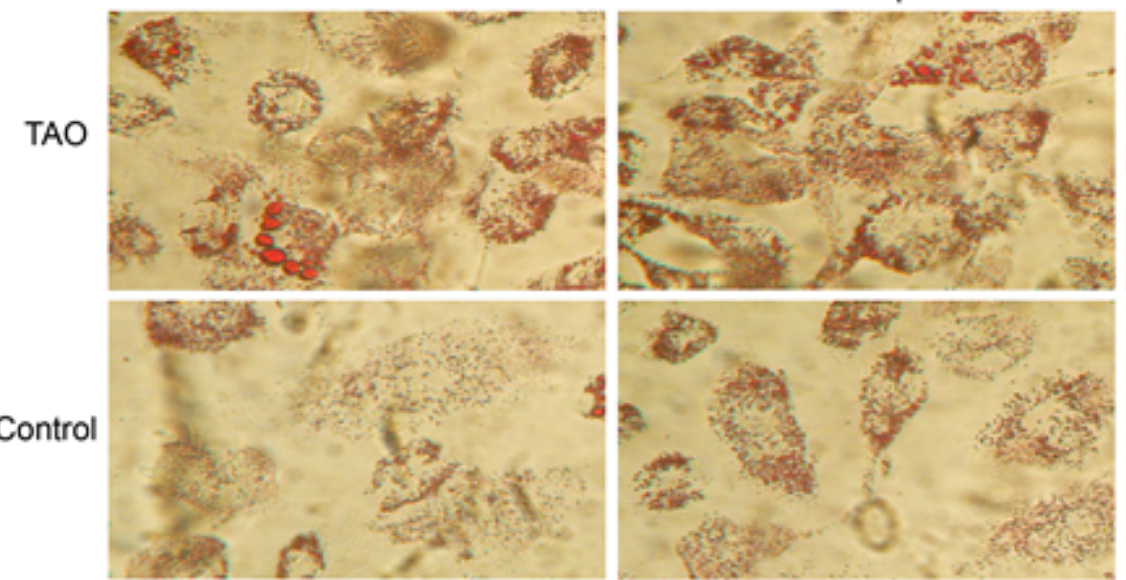

IL-6
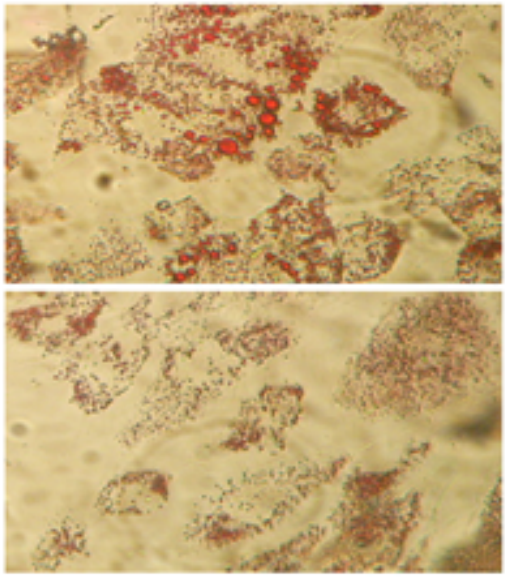

D
B

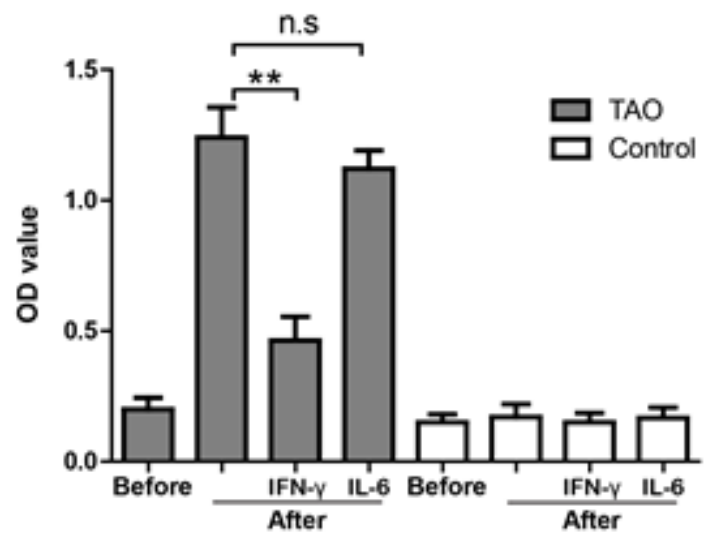

C

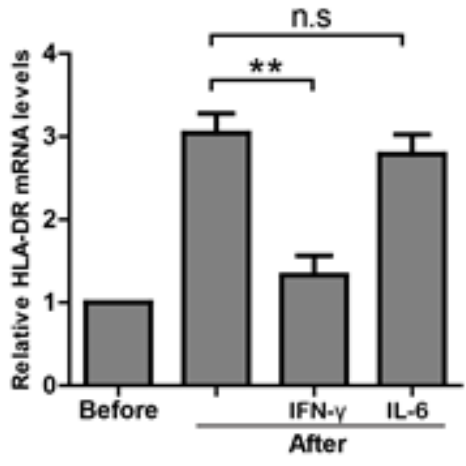

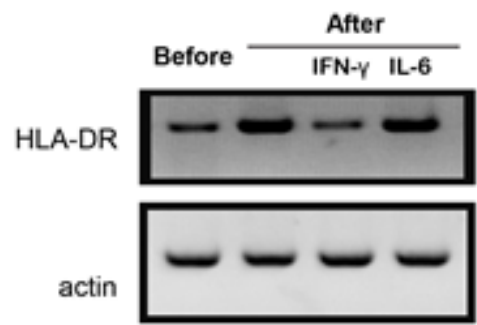

\section{Figure 3}

Effects of IFN-y or IL-6 in preadipocytes from TAO patients and controls (A) Oil Red O staining of differentiated preadipocytes from TAO patients and controls treated with IFN- $y$ or IL-6. (B) Intracytoplasmic lipid accumulation in preadipocytes from TAO patients and controls treated with IFN- $Y$ or IL-6. (C) Real time-PCR showing the HLA-DR mRNA level in preadipocytes from TAO patients treated with IFN- $\gamma$ or IL-6. (D) Immunoblot showing the HLA-DR protein level in preadipocytes from TAO patients treated with IFN-y or IL-6. 
A

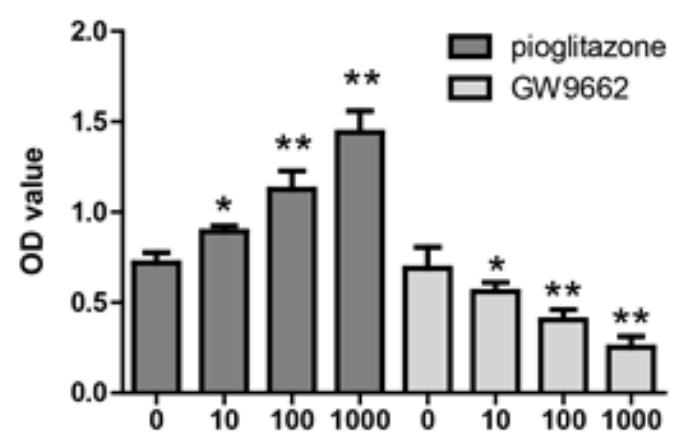

C

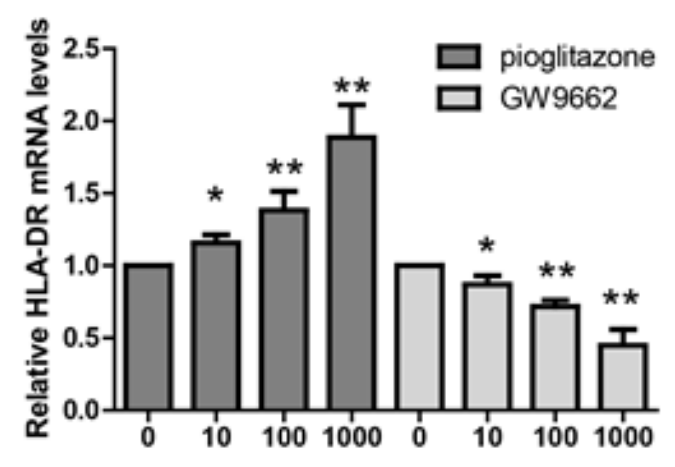

B

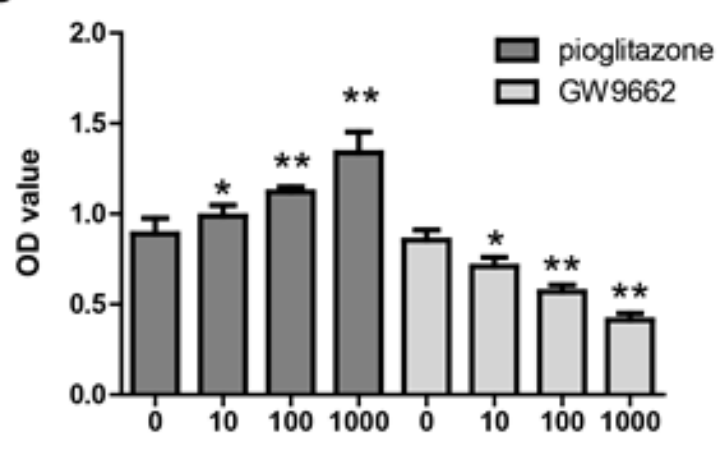

D
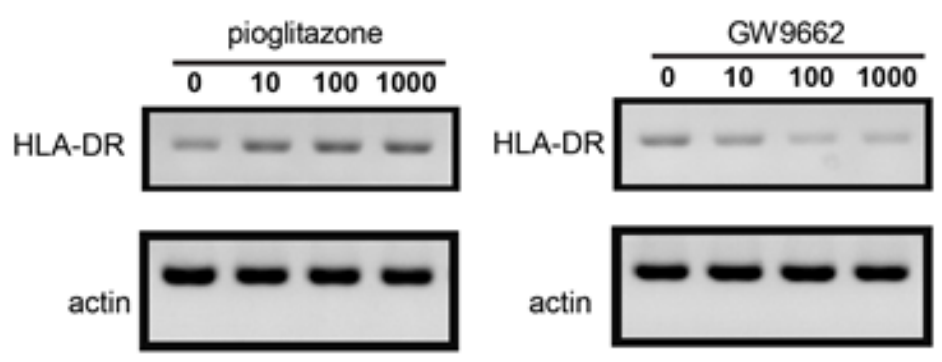

Figure 4

Effects of pioglitazone or GW9662 in TAO preadipocytes (A) MTT assay showing cell proliferation of preadipocytes from TAO patients treated with pioglitazone or GW9662 at indicated concentrations. (B) Intracytoplasmic lipid accumulation in preadipocytes from TAO patients treated with pioglitazone or GW9662 at indicated concentrations. (C) Real time-PCR showing the HLA-DR mRNA level in preadipocytes from TAO patients treated with pioglitazone or GW9662 at indicated concentrations. (D) Immunoblot showing the HLA-DR protein level in preadipocytes from TAO patients treated with pioglitazone or GW9662 at indicated concentrations. 